\title{
Bi-level optimization based on fuzzy if-then rule
}

\author{
Vishnu Pratap Singh ${ }^{1, *}$, Debjani Chakraborty ${ }^{2}$ \\ ${ }^{1}$ Department of Mathematics, Visvesvaraya National Institute of Technology Nagpur \\ Maharashtra 440010, Nagpur, India \\ E-mail: 〈vpsingh@mth.vnit.ac.in〉 \\ ${ }^{2}$ Department of Mathematics, Indian Institute of Technology Kharagpur \\ West Bengal 721302, Kharagpur, India \\ E-mail: 〈debjani@maths.iitkgp.ernet.in〉
}

\begin{abstract}
A bi-level programming problem has been developed where the functional relationship linking decision variables and the objective functions of leader and follower are not utterly well known to us. Because of the uncertainty in practical life decision-making situation most of the time it is inconvenient to find the veracious relationship between the objective functions of leader, follower and the decision variables. It is expected that the source of information which gives some command about the objective functions of leader and follower, is composed by a block of fuzzy if-then rules. In order to analyze the model, A dynamic programming approach with a suitable fuzzy reasoning scheme is applied to calculate the deterministic functional relationship linking the decision variables and the objective functions of leader as well as follower. Thus a bi-level programming problem is constructed from the actual fuzzy rule-based to the conventional bi-level programming problem. To solve the final problem, we use the lingo software to find the optimal of objective function of follower first and using its solution we optimize the objective function of leader. A numerical example has been solved to signify the computational procedure.
\end{abstract}

Keywords: bi-level programming, dynamic programming, fuzzy inference schemes, fuzzy rule-base

Received: August 19, 2019; accepted: October 31, 2019; available online: December 13, 2019

DOI: $10.17535 /$ crorr.2019.0025

\section{Introduction}

This paper contains a bi-level optimization problem and provide a fuzzy rule base structure of this hierarchical class of optimization problem. In classical optimization problem, bi-level programming problem (BLPP) has been used in a vast domain of practice. In the fields of management, it has been used to deal with facility location, environmental regulation, energy policy, etc. In the fields of economic planning, it has been used to deal with oil production, electric power pricing etc. In engineering, to solve optimal design, shape and structure. Decisionmakers (DMs) often deal with conflicting objectives in a hierarchical administrative structure. A decision maker has his own objective and decision space at one level and due to other level of hierarchy it may be influenced by the choice of other decision maker. There are two levels with two decision makers in bi-level programming problem. Decision makers of both level controls the variables of its own level. The DM of upper level is called leader and by his decision, the objective function of other level may be affected. Decision maker of lower level is called the follower. Decision makers of both level wants to optimize their objective function with the restriction of decision of one another. The hierarchical structure of the final problem needed

${ }^{*}$ Corresponding author. 
an optimal to the follower's problem first than a solution to the leader's problem is feasible and then the optimal is selected. Bi-level programming's solution is slightly difficult to deal because of its built-in non-convexity. The major segment of research in bi-level programming problem is still concerned on the deterministic case. BLPP were initially considered by Candler et. al. [6] and Fortuny et. al. [2] as a two player game where the first player can affect the resources available to a second player, this game is known as stackleberg game. For a given move of first player, the other player will maximize a linear program, subject to the available resources.

BLPP $[4,5,6]$ can be formulated as:

$$
\begin{aligned}
& \max _{x} F_{l}(x, y)=a x+b y \quad \text { where } y \text { solves } \\
& \max _{y} F_{f}(x, y)=c x+d y \\
& \text { s.t } \quad A x+B y \leq r .
\end{aligned}
$$

Here $a, c \in \mathbb{R}^{n_{1}}, b, d \in \mathbb{R}^{n_{2}}, r \in \mathbb{R}^{m}, A$ is an $m \times n_{1}$, matrix, $B$ is an $m \times n_{2}$ matrix. $(x, y) \in \mathbb{R}^{n}$ is a vector of decisions which can be controlled by the decision makers. $x \in \mathbb{R}^{n_{1}}$ is a vector control by leader and $y \in \mathbb{R}^{n_{2}}$ by follower, where $n_{1}+n_{2}=n . \quad F_{l}$ denotes the objectives functions of leader while $F_{f}$ denotes the objectives functions of the follower.

In the past, different approaches have been developed by Bard [3, 4], Bialas and Wen and Hsu $[17,18]$, Karwan [5] and others to solve deterministic bi-level programming problem. However, in real world situations, uncertainty and impreciseness are involved in defining the parameters and to form a mathematical model. It is difficult to define the imprecise and uncertain parameters in the objective functions and constraints in this case. Both the decision-makers need to take decision even if they do not know the parameter of the problem with full certainty hence bi-level programming problem with fuzzy parameter and Stochastic bi-level programming problem has been introduced. Sakawa et. al. [12] designed the bi-level programming problem with fuzzy variable and introduced a fuzzy programming method to solve it. Zang and $\mathrm{Lu}[13]$ has given a fuzzy number based Kuhn-Tucker condition to solve bi-level programming problem with fuzzy variable. Some multi-objective bi-level programming problem also has been investigated with fuzzy parameter [8,21]. For the randomness, Nishizaki et. al [11] introduced the two-level programming problem with parameters which are random variable. To solve this, they considered the variance of the objective function of leader (DM) and means of the objective function of the follower (DM) to find the deterministic programming problem. [22] solved large-scale 0-1 knapsack problem by the social-spider optimisation algorithm. Stochastic two-level programming problem has been investigated by Kosuch et al. [10] with probabilistic knapsack constraints, which can be used to jointly optimize service pricing and network resources. [20] article proposes a decision-making support called DMS4BPO that helps organizations in outsourcing their BPsto the Cloud by considering security, compliance, cost and performance criteria. DMS4BPO includes on the one hand an AHP-based method for the Cloud offer selection, and on the other hand, a Business Intelligence-based method for the exploitation of the execution history. [1] studied an inventory classification policy is provided. At each level, the loss profit of frequent items is determined. The obtained loss profit is used to rank frequent items at each level with respect to their category, content and brand. This helps inventory manager to determine the most profitable item with respect to their category, content and brand. [15] and [14] described the fuzzy randomness in bi-level programming problem using fuzzy random variable coefficients. [9] examines the discrepancies and analogies in addressing the collaborative decision making in two scientific fields: artificial intelligence and engineering design.

In this work, a mathematical tool based on fuzzy rule base is used to build a bi-level programming problem in a hierarchical administrative system. The antecedent part of the rule is defined as fuzzy inputs and its consequent part is a linear input output relation. The problem 
has been formulated for a bi-level system where a leader and a follower both deals with there own decision space but the functional relationship between the objectives and decision space has not been known exactly due to the imprecise nature of information they have. Most of the fuzzy bi-level programming problem may be modeled mathematically to find the solution which optimize the objective functions of leader as well as of follower. The decision space may contain $\widetilde{C} x \leq \widetilde{d}$ type of fuzzy inequality, where $\widetilde{C}, \widetilde{d}$ are fuzzy parameter and the objectives may be demonstrated as functions of decision variables. However, the information which is available may not be sufficient to form such type of model. In this situation, we can only form a linguistical relation between leader, follower's objective functions and the constraints from the past data. Chakraborty and Guha [7] formed a multi-objective optimization model in which they have used fuzzy rule base to define the exact relationship between the objective function and constraints. In this work, we have defined a bi-level programming problem with fuzzy rule base in which the exact relationship between the objective functions and the constraints has been defined from the past imprecise information to develop a real life hierarchical model. In designing such type of programming problem, the problem emerges as the functional connection between the objective functions and constraints can not be found directly in the given information. The framework has been defined linguistically to tackle such type of problem. It is expected that source of information from where some command may be acquired about the objective functions of leader and follower which is composed by a block of fuzzy if-then rules. At both the level of model, we have fuzzy if-then rules that connects the objective functions and constraints. At first level, the leader's fuzzy rules are defined and at second level the follower's fuzzy rules are defined. To solve the problem, we have developed a dynamic programming approach using Takagi and Sugeno fuzzy reasoning scheme [16] to solve the problem.

The paper is organized as follows. Linguistic variable and fuzzy reasoning scheme has been defined in Section 2. Next, in Section 3, fuzzy rule-base bi-level programming problem and methodology of solving this problem is considered. Section 4 contains a numerical example and the conclusion has been given in Section 5.

\section{Preliminaries}

\subsection{Linguistic variable}

In our natural or real life language, linguistic variable are those variable which are real life words or sentences instead of crisp numbers [19]. Each value of the linguistic variable can be represented by using triangular fuzzy number which are defined with the assist of membership functions. In particular, a linguistic variable can be evaluated either as a value of a fuzzy number or values which are defined in linguistic terms.

Definition 1. (Linguistic variable): A quintuple ( $y, \Im(y), Y, G, M)$ denotes a linguistic variable. Here $y$ denotes the linguistic variable's name; $\Im(y)$ denotes the term set of $y$, i.e., the set of names of linguistic values of $y$ with each value being a fuzzy number defined on $Y$; $M$ is a semantic rule for associating with each value its meaning and $G$ is a syntactic rule for developing the names of values of $x . \Im(Y)$ denotes the family of all fuzzy sets in $Y$.

Here, it is considered that the values of each term of the linguistic variables are defined in the interval $[c, d] \subset \mathbb{R}$. Let $Y=[c, d]$ and $\Im(y)$ consists of $p+1,(P \geq 2)$, terms as given in the Figure 1,

$$
\Im(y)=\left\{\operatorname{low}_{1}, \operatorname{around}(c+\beta), \operatorname{around}(c+2 \beta), \ldots, \operatorname{around}(c+(p-1) \beta), \operatorname{high}_{P}\right\},
$$

where $\beta=(d-c) / p$.

Here each value is defined as a triangular fuzzy number. It may be illustrated by the membership functions $\mu_{\widetilde{B}_{1}}, \ldots, \mu_{\widetilde{B}_{P+1}}$ of triangular fuzzy number of the following form: 


$$
\begin{aligned}
& \mu_{\widetilde{B}_{1}}(y)=\mu_{\text {low }_{1}}(y) \\
& \mu_{\widetilde{B}_{1}}(y)= \begin{cases}1-(y-c) /(d-c), & \text { for } c \leq y \leq d \\
0, & \text { otherwise }\end{cases}
\end{aligned}
$$

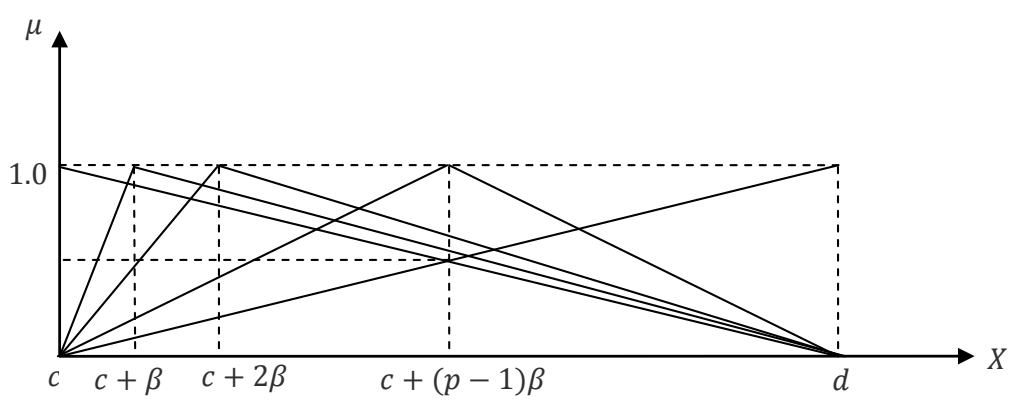

Figure 1: Membership value for the linguistic variable with $(P+1)$ terms

The fuzzy number $\widetilde{B}_{1}$ is denoted by $\widetilde{B}_{1}=(c ; 0, d-c)$.

$$
\begin{aligned}
& \mu_{\widetilde{B}_{p}}(y)=\mu_{\text {around }(c+p \beta)}(y) \\
& \mu_{\widetilde{B}_{p}}(y)= \begin{cases}1-(c+p \beta-y) / p \beta, & \text { for } c \leq y \leq c+p \beta \\
1-(y-c-p \beta) /(d-c-p \beta), & \text { for } c+p \beta \leq y \leq d \\
0, & \text { otherwise }\end{cases}
\end{aligned}
$$

for $1 \leq p \leq(P-1)$ and each of the corresponding fuzzy number $\widetilde{B}_{k}$ is denoted as $\widetilde{B}_{k}=$ $(c+p \beta ; p \beta, d-c-p \beta)$.

$$
\begin{aligned}
& \mu_{\widetilde{B}_{P+1}}(y)=\mu_{\text {igh }_{P}}(y) \\
& \mu_{\widetilde{B}_{P+1}}(y)= \begin{cases}1-(d-y) /(d-c), & \text { for } c \leq y \leq d \\
0, & \text { otherwise }\end{cases}
\end{aligned}
$$

The triangular fuzzy number $\widetilde{B}_{P+1}$ is denoted by $\widetilde{B}_{P+1}=(d ; d-c, 0)$. In this work, to represent a triangular fuzzy number in $[0,1]$ by its membership function, the standard notation, $\widetilde{B}=(m ; \alpha, \beta)$ is used. Where $m$ represents the middle value $\alpha$ represents the left spread and $\beta$ represents the right spread. When a very little knowledge is known about the boundaries of each value, each value is extended over the complete domain through the middle values of each of the fuzzy number which are located at a fixed distance apart.

Example 1. Suppose the amount of water during flood in a particular region is interpreted as a linguistic variable, and then its term set is of the following type:

$$
\Im(\text { Amount of water })=\{\text { very low, low, medium, high, veryhigh }\}
$$

Where each term in $\Im$ (amount of water) is characterized by a fuzzy set in the universe of discourse [0,100]. Then, using the fuzzy partition given in (4), (5) and (6) for the interval [0,100], each term can be transformed to associated fuzzy numbers as provided in Table 1. 


\begin{tabular}{|c|c|c|}
\hline$\Im\left(y_{3}\right)$ & Fuzzy numbers & Normalized fuzzy numbers \\
\hline Very Low (VL) & $\left(\begin{array}{lll}0, & 0, & 100)\end{array}\right.$ & $\left(\begin{array}{lll}0, & 0, & 1\end{array}\right)$ \\
\hline Low $(\mathrm{L})$ & 75) & $(0.25$ \\
\hline Medium (M) & $(50$, & $0.5)$ \\
\hline $\operatorname{High}(\mathrm{H})$ & $75,25)$ & $(0.75$ \\
\hline Very High (VH) & $(100,100,0)$ & $(1$, \\
\hline
\end{tabular}

Table 1: The term set $\Im$ (amount of water)

\subsection{Fuzzy inference schemes}

Fuzzy Mathematicians have been introduced different types of fuzzy reasoning schemes. The distinction between different reasoning schemes consist in the illustration of the consequents of theirs fuzzy implication. A fuzzy if- then rule base system has been developed so that it joins the input variables to the output variable by means of if - then rules. For some given term of the input values, the degree of fulfillment of a rule is acquired by grouping the membership functions of these input values into the respective fuzzy number. The output is obtained by the degrees of fulfillment and the consequence of the implications. Several fuzzy inference schemes are available in the literature. In this work, to make a decision from fuzzy if - then rule based system, we have used Takagi and Sugeno [16] fuzzy inference scheme, which is given below.

\subsubsection{Sugeno and Takagi fuzzy reasoning scheme}

$\Re_{1}$ : If $r_{1}$ is $\widetilde{B}_{11}$ and $r_{2}$ is $\widetilde{B}_{12}$ and $\ldots$ and $r_{n}$ is $\widetilde{B}_{1 n}$ then $z=b_{11} r_{1}+b_{12} r_{2}+\cdots+b_{1 n} r_{n}+c_{1}$

$\Re_{2}$ : If $r_{1}$ is $\widetilde{B}_{21}$ and $r_{2}$ is $\widetilde{B}_{22}$ and $\ldots$ and $r_{n}$ is $\widetilde{B}_{2 n}$ then $z=b_{21} r_{1}+b_{22} r_{2}+\cdots+b_{2 n} r_{n}+c_{2}$

$\Re_{p}$ : If $r_{1}$ is $\widetilde{B}_{p 1}$ and $r_{2}$ is $\widetilde{B}_{p 2}$ and $\ldots$ and $r_{n}$ is $\widetilde{B}_{p n}$ then $z=b_{p 1} r_{1}+b_{p 2} r_{2}+\cdots+b_{p n} r_{n}+c_{p}$ Input: $r_{1}$ is $\bar{y}_{1}$ and $r_{2}$ is $\bar{y}_{2}$ and $\ldots$ and $r_{n}$ is $\bar{y}_{n}$

Output: $\quad z$ is $z_{T g S}$,

where $\widetilde{B}_{j k} \in \Im\left\{R_{k}\right\}$ is the term set of the linguistic variable $r_{k}$ which has been defined in the universe of discourse $R_{k} \subset \mathbb{R}$, and $b_{j k}$ and $c_{j}$ are real numbers for $j=1,2, \ldots, p$ and $k=1,2, \ldots, n$. The methodology for find the deterministic output $z_{T g S}$, from the crisp input vector $y=\left\{y_{1}, y_{2}, \ldots, y_{n}\right\}$ and fuzzy impications $\left\{\Re_{1}, \Re_{2}, \ldots, \Re_{p}\right\}$ are given below:

The degree to which input matches the $j^{\text {th }}$ rule $\Re_{j}$ is typically computed using the relation

$$
\begin{array}{r}
l_{j}=T_{p}\left(\mu_{\widetilde{B}_{j 1}}\left(y_{1}\right), \mu_{\widetilde{B}_{j 2}}\left(y_{2}\right), \ldots, \mu_{\widetilde{B}_{j n}}\left(y_{n}\right)\right), \\
\text { for } j=1,2, \ldots, p .
\end{array}
$$

Then the outputs of the individual rule calculated from the implications

$$
z_{j}(y)=\sum_{k=1}^{n} b_{j k} y_{k}+c_{j}
$$

Finally, the output of the fuzzy reasoning scheme is calculated by the following expression

$$
z_{T g S}=\frac{l_{1} z_{1}+l_{2} z_{2}+\cdots+l_{p} z_{p}}{l_{1}+l_{2}+\cdots+l_{p}}
$$




\section{Fuzzy rule-based bi-level programming problem}

In a two level administrative structure, to built a mathematical model we need deterministic type information. Where both the decision maker have their own objective function to optimize under some crisp inequalities. If the information source from where the crisp inequalities has to be built, is not known precisely i.e we know the linguistic relationship between the objective function and the constraints. In that case it is difficult to build a deterministic bi-level programming model. Here, we present a fuzzy rule-base bi-level programming model which overcomes this difficulty. The model is represented is as follows:

$$
\begin{aligned}
& \widetilde{\max _{x}} \widetilde{f}_{l}(x, y) \text { where } y \text { solves } \\
& \underset{\max _{y}}{ } \widetilde{f}_{f}(x, y) \\
& \quad \text { s.t. }\left\{\Re_{1}(x, y), \Re_{2}(x, y), \ldots, \Re_{p}(x, y) \mid x, y \in \widetilde{R} \subset \mathbb{R}^{m}\right\},
\end{aligned}
$$

$\widetilde{f}_{l}(x, y)$ and $\widetilde{f}_{f}(x, y)$ are the leader and followers' objective functions respectively, $x$ is the linguistic variable controlled by leader and $y$ is the linguistic variable controlled by follower.

Depending upon the nature of the information available for the objective functions of leader and follower, we can write our problem as follows:

$\Re_{j}(x, y)$ : If $x_{1}$ is $\widetilde{A}_{j 1}$ and $x_{2}$ is $\widetilde{A}_{j 2} \ldots x_{m}$ is $\widetilde{A}_{j m}$ and $y_{1}$ is $\widetilde{B}_{j 1}$ and $y_{2}$ is $\widetilde{B}_{j 2} \ldots y_{m}$ is $\widetilde{B}_{j m}$ then $\widetilde{f}_{l j}(x, y)=\sum_{k=1}^{m} a_{j k} x_{k}+\sum_{k=1}^{m} b_{j k} y_{k}$.

where $y$ solves

$\Re_{j}(y)$ : If $x_{1}$ is $\widetilde{A}_{j 1}$ and $x_{2}$ is $\widetilde{A}_{j 2} \ldots x_{m}$ is $\widetilde{A}_{j m}$ and $y_{1}$ is $\widetilde{B}_{j 1}$ and $y_{2}$ is $\widetilde{B}_{j 2} \ldots y_{m}$ is $\widetilde{B}_{j m}$ then $\tilde{f}_{f j}(X, Y)=\sum_{k=1}^{m} c_{j k} x_{k}+\sum_{k=1}^{m} d_{j k} y_{k}$.

for $j=1,2, \ldots, p$.

After Normalization, the fuzzy model takes the form as follows:

$\Re_{j}(x)$ : If $x_{1}$ is $\widetilde{N A}_{j 1}$ and $x_{2}$ is $\widetilde{N A}_{j 2} \ldots x_{m}$ is $\widetilde{N A}_{j m}$ and $y_{1}$ is $\widetilde{N B}_{j 1}$ and $y_{2}$ is $\widetilde{N B}_{j 2} \ldots y_{m}$ is $\widetilde{N B}_{j m}$ then $\widetilde{f}_{l j}(x, y)=\sum_{k=1}^{m} a_{j k} x_{k}+\sum_{k=1}^{m} b_{j k} y_{k}$.

where $y$ solves

$\Re_{j}(y)$ : If $x_{1}$ is $\widetilde{N A}_{j 1}$ and $x_{2}$ is $\widetilde{N A}_{j 2} \ldots x_{m}$ is $\widetilde{N A}_{j m}$ and $y_{1}$ is $\widetilde{N B}_{j 1}$ and $y_{2}$ is $\widetilde{N B}_{j 2} \ldots y_{m}$ is $\widetilde{N B}_{j m}$ then $\widetilde{f}_{f j}(X, Y)=\sum_{k=1}^{m} c_{j k} x_{k}+\sum_{k=1}^{m} d_{j k} y_{k}$.

for $j=1,2, \ldots, p$.

\subsection{Stage-wise decomposition}

In two-level programming problem, the feasible solution of first level is acceptable only if it is the optimal solution of the second level problem. To find the optimal solution of both the level, we can update the above $n$-stage bi-level programming problem in which the constraints are the fuzzy rule of both the decision makers i.e it contains the variable which is controlled by leader and the variable which is controlled by follower. 


\subsection{Stage-wise computational procedure of fuzzy rule-base bi-level programming problem}

Here, we have taken the superscript in each notation to denote the stage number.

\section{Stage 1 :}

Leader's Fuzzy rule:

$\Re_{j}^{1}\left(x_{1}, y_{1}\right)$ : If $x_{1}$ is $\widetilde{N A}_{j 1}$ and $y_{1}$ is $\widetilde{N B}_{j 1}$ then $\widetilde{f}_{l j}^{1}\left(x_{1}, y_{1}\right)=a_{j 1} x_{1}+b_{j 1} y_{1}, j=1,2, \ldots, p$ where $\mathrm{Y}$ solves

Follower's fuzzy rule :

$\Re_{j}^{1}\left(x_{1}, y_{1}\right):$ If $x_{1}$ is $\widetilde{N A}_{j 1}$ and $y_{1}$ is $\widetilde{N B}_{j 1}$ then $\widetilde{f}_{f j}^{1}\left(x_{1}, y_{1}\right)=c_{j 1} x_{1}+d_{j 1} y_{1}, j=1,2, \ldots, p$

For solving above fuzzy bi-level programming model. First, we consider the follower's problem:

Follower's fuzzy rule :

$\Re_{j}^{1}\left(x_{1}, y_{1}\right)$ : If $x_{1}$ is $\widetilde{N A}_{j 1}$ and $y_{1}$ is $\widetilde{N B}_{j 1}$ then $\widetilde{f}_{f j}^{1}\left(x_{1}, y_{1}\right)=c_{j 1} x_{1}+d_{j 1} y_{1}, j=1,2, \ldots, p$

We take $x_{c 1}$ and $y_{c 1}$ as crisp input corresponding to $x_{1}$ and $y_{1}$. The membership degree up to which the $j^{\text {th }}$ rule $\Re_{j}^{1}\left(x_{1}, y_{1}\right)$ matches the given input, is calculated by the following expression:

$$
l_{j}^{1}=T\left(\mu_{\widetilde{N A}_{j 1}}\left(x_{1}\right), \mu_{\widetilde{N B}}\left(y_{1}\right)\right)
$$

Objective functions of the follower for the individual rule are given by

$$
f_{f j}^{1}\left(x_{c 1}, y_{c 1}\right)=c_{j 1} x_{c 1}+d_{j 1} y_{c 1}
$$

The crisp objective function for the follower's fuzzy model has been given by

$$
F_{f}^{1}\left(x_{c 1}, y_{c 1}\right)=\frac{l_{1} f_{f 1}^{1}+l_{2} f_{f 2}^{1}+\cdots+l_{p} f_{f p}^{1}}{l_{1}+l_{2}+\cdots+l_{p}}
$$

The follower's objective function is the piece-wise continuous function in the interval $x_{c 1}, y_{c 1} \in$ $[0,1]$. To calculate the follower's optimal solution under the optimal of leader, we calculate the optimal of this function with general value of $0<y_{c 1}<=x_{c 1}$ for each sub interval in which the function is defined.

We use the value of optimal $y_{c 1}^{*}$ in the leader's objective function. To calculate the leader's crisp objective function, we follow the same steps after putting the value $y_{c 1}=y_{c 1}^{*}$.

Objective functions of the leader for the individual rule are given by

$$
f_{l j}^{1}\left(x_{c 1}, y_{c 1}^{*}\right)=c_{j 1} x_{c 1}+d_{j 1} y_{c 1}^{*}
$$

Leader's objective function will be of the form

$$
F_{l}^{1}\left(x_{c 1}\right)=\frac{l_{1} f_{l 1}^{1}+l_{2} f_{l 2}^{1}+\cdots+l_{p} f_{l p}^{1}}{l_{1}+l_{2}+\cdots+l_{p}}
$$


To solve the second stage problem, we need to define a recurrence relation to connect the two stages, which satisfies the principle of optimality. The leader's and follower's recurrence relation has been given by:

Follower's recurrence relation:

$$
F_{f}^{r}\left(x_{c r}, y_{c r}\right)=F_{f}^{r-1}\left(x_{c r-1}^{*}, y_{c r-1}^{*}\right)+\frac{l_{1} f_{f 1}^{r}+l_{2} f_{f 2}^{r}+\cdots+l_{p} f_{f p}^{r}}{l_{1}+l_{2}+\cdots+l_{p}}
$$

Leader's recurrence relation

$$
F_{l}^{r}\left(x_{c r}\right)=F_{l}^{r-1}\left(x_{c r-1}^{*}\right)+\frac{l_{1} f_{l 1}^{r}+l_{2} f_{l 2}^{r}+\cdots+l_{p} f_{l p}^{r}}{l_{1}+l_{2}+\cdots+l_{p}}
$$

To solve the $r^{\text {th }}-$ stage problem:

Leader's Fuzzy rule:

$\Re_{j}^{r}\left(x_{r}, y_{r}\right):$ If $x_{1}$ is $\widetilde{N A}_{j r}$ and $y_{1}$ is $\widetilde{N B}_{j r}$ then $\widetilde{f}_{l j}^{r}\left(x_{r}, y_{r}\right)=a_{j r} x_{r}+b_{j r} y_{r}, j=1,2, \ldots, p$ where $\mathrm{Y}$ solves

Follower's fuzzy rule :

$\Re_{j}^{r}\left(x_{r}, y_{r}\right):$ If $x_{1}$ is $\widetilde{N A}_{j r}$ and $y_{1}$ is $\widetilde{N B}_{j r}$ then $\widetilde{f}_{f j}^{1}\left(x_{r}, y_{r}\right)=c_{j r} x_{r}+d_{j r} y_{r}, j=1,2, \ldots, p$

For solving above fuzzy bi-level programming model. First, we consider the follower's problem:

Follower's fuzzy rule :

$\Re_{j}^{r}\left(x_{r}, y_{r}\right):$ If $x_{r}$ is $\widetilde{N A}_{j r}$ and $y_{r}$ is $\widetilde{N B}_{j r}$ then $\widetilde{f}_{f j}^{r}\left(x_{r}, y_{r}\right)=c_{j r} x_{r}+d_{j r} y_{r}, j=1,2, \ldots, p$

We take $x_{c r}$ and $y_{c r}$ as crisp input corresponding to $x_{r}$ and $y_{r}$. The membership degree up to which the $j^{\text {th }}$ rule $\Re_{j}^{1}\left(x_{1}, y_{1}\right)$ matches the given input, is calculated by the following expression:

$$
l_{j}^{r}=T\left(\mu_{\widetilde{N A}_{j r}}\left(x_{r}\right), \mu_{\widetilde{N B}_{j r}}\left(y_{r}\right)\right)
$$

Objective functions of the follower for the individual rule are given by

$$
f_{f j}^{r}\left(x_{c r}, y_{c r}\right)=c_{j r} x_{c r}+d_{j r} y_{c r}
$$

The crisp objective function for the follower's fuzzy model has been given by

$$
F_{f}^{r}\left(x_{c r}, y_{c r}\right)=F_{f}^{r-1}\left(x_{c r-1}^{*}, y_{c r-1}^{*}\right)+\frac{l_{1} f_{f 1}^{r}+l_{2} f_{f 2}^{r}+\cdots+l_{p} f_{f p}^{r}}{l_{1}+l_{2}+\cdots+l_{p}}
$$

The follower's objective function is the piece-wise continuous function in the interval $x_{c r}, y_{c r} \in$ $[0,1]$. To calculate the follower's optimal under the optimal of leader, we calculate the optimal of this function with general value of $0<=x<=y$ for each sub interval in which the function is defined. 
We use the value of optimal $y_{c r}^{*}$ in the leader's objective function. To calculate the leader's crisp objective function, we follow the same steps by putting the value $y_{c r}=y_{c r}^{*}$

Objective functions of the leader for the individual rule are given by

$$
f_{l j}^{r}\left(x_{c r}, y_{c r}^{*}\right)=c_{j r} x_{c r}+d_{j r} y_{c r}^{*}
$$

Leader's objective function will be of the form

$$
F_{l}^{r}\left(x_{c r}\right)=F_{l}^{r-1}\left(x_{c r-1}^{*}\right)+\frac{l_{1} f_{l 1}^{r}+l_{2} f_{l 2}^{r}+\cdots+l_{p} f_{l p}^{r}}{l_{1}+l_{2}+\cdots+l_{p}}
$$

To find the optimal solution of the fuzzy bi-level model, We proceed in a similar manner up to $n^{\text {th }}$-stage. At last stage, The optimal solution contains the optimal return from each stage.

\subsection{Algorithm}

Step 1 Determine the normalized fuzzy number of each linguistic variable which represents the linguistic relationship between the decision variables and objectives of leader's and follower's.

Step 2 Convert the $n$ variable problem into $n$ stage single variable problem for both the decision makers.

Step 3 At $r^{\text {th }}$ stage (for $r=1,2, \ldots, m$ ), calculate the deterministic functional relationship of decision variable and the follower's objective function then use the recurrence relation of follower to calculate the optimal return at $r^{t h}$ stage in the interval $0 \leq y \leq x$, which incorporates the optimal return from the previous stage. The recurrence relation of the follower's problem is given by

$$
F_{f}^{r}\left(x_{c r}, y_{c r}\right)=F_{f}^{r-1}\left(x_{c r-1}^{*}, y_{c r-1}^{*}\right)+\frac{l_{1} f_{f 1}^{r}+l_{2} f_{f 2}^{r}+\cdots+l_{p} f_{f p}^{r}}{l_{1}+l_{2}+\cdots+l_{p}}
$$

where $F_{f}^{r-1}\left(x_{c r-1}^{*}, y_{c r-1}^{*}\right)=0$ for $r=1$.

Step 4 Calculate the deterministic functional relationship of decision variable and the leader's objective function then use the recurrence relation of leader to calculate the optimal return at $r^{t h}$ stage, which incorporates the optimal return from the previous stage. The recurrence relation of the leader's problem is given by

$$
F_{l}^{r}\left(x_{c r}\right)=F_{l}^{r-1}\left(x_{c r-1}^{*}\right)+\frac{l_{1} f_{l 1}^{r}+l_{2} f_{l 2}^{r}+\cdots+l_{p} f_{l p}^{r}}{l_{1}+l_{2}+\cdots+l_{p}}
$$

where $F_{l}^{r-1}\left(x_{c r-1}^{*}\right)=0$ for $r=1$.

Step 5 Use the steps 3,4 to find the optimal solution for leader and follower objective functions at $r^{t h}$ stage which should be incorporate to the objective functions at $(r+1)^{t h}$ stage. Repeat the process for $r=1,2, \ldots, m$.

Step 6 Stop when $r=m$. 


\section{Numerical example}

Let us consider the fuzzy rule-based bi-level programming problem where the leader and follower rules are defined linguistically as follows:

Leader's fuzzy rules :

$\Re_{1}(x, y)$ : If $x_{1}$ is high and $x_{2}$ is very high and $y_{1}$ is medium and $y_{2}$ is low then $\widetilde{f}_{l}\left(x_{1}, x_{2}, y_{1}, y_{2}\right)=$ $-x_{1}+x_{2}+y_{1} / 2-y_{2}$

$\Re_{2}(x, y)$ : If $x_{1}$ is low and $x_{2}$ is high and $y_{1}$ is very low and $y_{2}$ is high then $\widetilde{f}_{l}\left(x_{1}, x_{2}, y_{1}, y_{2}\right)=$ $x_{1}+x_{2}+y_{1}+y_{2}$

where $Y=\left(y_{1}, y_{2}\right)$ solves the follower's fuzzy rules given by

$\Re_{1}(x, y)$ : If $x_{1}$ is high and $x_{2}$ is very high and $y_{1}$ is medium and $y_{2}$ is low then $\widetilde{f}_{f}\left(x_{1}, x_{2}, y_{1}, y_{2}\right)=$ $x_{1}+x_{2} / 2-y_{1}+y_{2}$

$\Re_{2}(x, y)$ : If $x_{1}$ is low and $x_{2}$ is high and $y_{1}$ is very low and $y_{2}$ is high then $\widetilde{f}_{f}\left(x_{1}, x_{2}, y_{1}, y_{2}\right)=$ $-x_{1}+x_{2}+y_{1}-y_{2}$

\begin{tabular}{|l|l|l||l|l|l|}
\hline$\Im\left(x_{1}\right)$ & $\begin{array}{l}\text { Fuzzy } \\
\text { numbers }\end{array}$ & $\begin{array}{l}\text { Normalized } \\
\text { fuzzy numbers }\end{array}$ & $\Im\left(x_{1}\right)$ & $\begin{array}{l}\text { Fuzzy } \\
\text { numbers }\end{array}$ & $\begin{array}{l}\text { Normalized } \\
\text { fuzzy numbers }\end{array}$ \\
\hline Very Low (VL) & $(, .0,10)$ & $(0,0,1.0)$ & Very Low (VL) & $(10,0,40)$ & $(0.2,0.2,0.8)$ \\
Low (L) & $(2.5,2.5,7.5)$ & $(0.25,0.25,0.75)$ & Low (L) & $(20,10,30)$ & $(0.4,0.2,0.6)$ \\
Medium (M) & $(5.0,5.0,5.0)$ & $(0.5,0.5,0.5)$ & Medium (M) & $(30,20,20)$ & $(0.6,0.4,0.4)$ \\
High (H) & $(7.5,7.5,2.5)$ & $(0.75,0.75,0.25)$ & High (H) & $(40,30,10)$ & $(0.8,0.6,0.2)$ \\
Very High (VH) & $(10.0,10.0,0)$ & $(1.0,1.0,0)$ & Very High (VH) & $(50,40,0)$ & $(1.0,0.8,0)$ \\
\hline
\end{tabular}

Table 1: Linguistic value for variable $x_{1} \quad$ Table 2: Linguistic value for variable $x_{2}$

\begin{tabular}{|l|l|l||l|l|l|}
\hline$\Im\left(y_{1}\right)$ & $\begin{array}{l}\text { Fuzzy } \\
\text { numbers }\end{array}$ & $\begin{array}{l}\text { Normalized } \\
\text { fuzzy numbers }\end{array}$ & $\Im\left(y_{2}\right)$ & $\begin{array}{l}\text { Fuzzy } \\
\text { numbers }\end{array}$ & $\begin{array}{l}\text { Normalized } \\
\text { fuzzy numbers }\end{array}$ \\
\hline Very Low (VL) & $(2,1,8)$ & $(0.2,0.1,0.8)$ & Very Low $(\mathrm{VL})$ & $(50,0,50)$ & $(0.2,0,0.2)$ \\
Low (L) & $(10,10,20)$ & $(0.33,0.33,0.67)$ & Low $(\mathrm{L})$ & $(60,10,40)$ & $(0.6,0.1,0.4)$ \\
Medium $(\mathrm{M})$ & $(20,10,30)$ & $(0.4,0.2,0.6)$ & Medium $(\mathrm{M})$ & $(70,20,30)$ & $(0.7,0.2,0.3)$ \\
High (H) & $(50,20,30)$ & $(0.63,0.25,0.34)$ & High $(\mathrm{H})$ & $(85,35,15)$ & $(0.85,0.35,0.15)$ \\
Very High (VH) & $(100,50,50)$ & $(0.67,0.33,0.33)$ & Very High $(\mathrm{VH})$ & $(100,50,0)$ & $(1,0.5,0)$ \\
\hline
\end{tabular}

Table 3: Linguistic value for variable $y_{1}$

Table 4: Linguistic value for variable $y_{2}$

First we calculate the normalized linguistic fuzzy number from the given linguistic variables which are given in the above tables. 
Stage 1 Problem:

Leader's fuzzy rules :

$\Re_{1}^{1}(x, y)$ : If $x_{1}$ is high and $y_{1}$ is medium then $\widetilde{f}_{l}^{1}\left(x_{1}, y_{1}\right)=-x_{1}+y_{1} / 2$

$\Re_{2}^{1}(x, y)$ : If $x_{1}$ is low and $y_{1}$ is very low then $\widetilde{f}_{l}^{1}\left(x_{1}, y_{1}\right)=x_{1}+y_{1}$

where $y_{1}$ solves the follower's fuzzy rules by

$\Re_{1}^{1}(x, y)$ : If $x_{1}$ is high and $y_{1}$ is medium then $\tilde{f}_{f}^{1}\left(x_{1}, y_{1}\right)=x_{1}-y_{1}$

$\Re_{2}^{1}(x, y)$ : If $x_{1}$ is low and $y_{1}$ is high then $\widetilde{f}_{f}^{1}\left(x_{1}, y_{1}\right)=-x_{1}+y_{1}$

First, we solve the follower's problem:

$$
\begin{gathered}
l_{1}^{1}= \begin{cases}\frac{x_{c 1}}{0.75} \cdot \frac{y_{c 1}-0.2}{0.2}, & \text { for } 0.2 \leq x_{c 1}, y_{c 1} \leq 0.4 \\
\frac{x_{c 1}}{0.75} \cdot \frac{1-y_{c 1}}{0.6}, & \text { for } 0.4 \leq x_{c 1}, y_{c 1} \leq 0.75 \\
\frac{1-x_{c 1}}{0.25} \cdot \frac{1-y_{c 1}}{0.6}, & \text { for } 0.75 \leq x_{c 1}, y_{c 1} \leq 1\end{cases} \\
l_{2}^{1}= \begin{cases}\frac{x_{c 1}}{0.25} \cdot \frac{y_{c 1}-0.1}{0.1}, & \text { for } 0.1 \leq x_{c 1}, y_{c 1} \leq 0.2 \\
\frac{x_{c 1}}{0.25} \cdot \frac{1-y_{c 1}}{0.8}, & \text { for } 0.2 \leq x_{c 1}, y_{c 1} \leq 0.25 \\
\frac{1-x_{c 1}}{0.75} \cdot \frac{1-y_{c 1}}{0.8}, & \text { for } 0.25 \leq x_{c 1}, y_{c 1} \leq 1\end{cases}
\end{gathered}
$$

Using our approach the follower's objective function can be calculated as:

$$
F_{f}^{1}\left(x_{c 1}, y_{c 1}\right)= \begin{cases}\frac{\left(\frac{x_{c 1}}{0.75}\right)\left(\frac{y_{c 1}-0.2}{0.2}\right)\left(x_{c 1}-y_{c 1}\right)+\left(\frac{x_{c 1}}{0.25}\right)\left(\frac{1-y_{c 1}}{0.8}\right)\left(y_{c 1}-x_{c 1}\right)}{\left(\frac{x_{c 1}}{0.75}\right)\left(\frac{y_{c 1}-0.2}{0.2}\right)+\left(\frac{x_{c 1}}{0.25}\right)\left(\frac{1-y_{c 1}}{0.8}\right)} & \text { for } 0.2 \leq y_{c 1} \leq 0.25 \\ \frac{\left(\frac{x_{c 1}}{0.75}\right)\left(\frac{y_{c 1}-0.2}{0.2}\right)\left(x_{c 1}-y_{c 1}\right)+\left(\frac{1-x_{c 1}}{0.75}\right)\left(\frac{1-y_{c 1}}{0.8}\right)\left(y_{c 1}-x_{c 1}\right)}{\left(\frac{x_{c 1}}{0.75}\right)\left(\frac{y_{c 1}-0.2}{0.2}\right)+\left(\frac{1-x_{c 1}}{0.75}\right)\left(\frac{1-y_{c 1}}{0.8}\right)} & \text { for } 0.25 \leq y_{c 1} \leq 0.4 \\ \frac{\left(\frac{x_{c 1}}{0.75}\right)\left(\frac{1-y_{c 1}}{0.6}\right)\left(x_{c 1}-y_{c 1}\right)+\left(\frac{1-x_{c 1}}{0.75}\right)\left(\frac{1-y_{c 1}}{0.8}\right)\left(y_{c 1}-x_{c 1}\right)}{\left(\frac{x_{c 1}}{0.75}\right)\left(\frac{1-y_{c 1}}{0.6}\right)+\left(\frac{1-x_{c 1}}{0.75}\right)\left(\frac{1-y_{c 1}}{0.8}\right)} & \text { for } 0.4 \leq y_{c 1} \leq 0.75 \\ \frac{\left(\frac{1-x_{c 1}}{0.25}\right)\left(\frac{1-y_{c 1}}{0.6}\right)\left(x_{c 1}-y_{c 1}\right)+\left(\frac{1-x_{c 1}}{0.75}\right)\left(\frac{1-y_{c 1}}{0.8}\right)\left(y_{c 1}-x_{c 1}\right)}{\left(\frac{1-x_{c 1}}{0.25}\right)\left(\frac{1-y_{c 1}}{0.6}\right)+\left(\frac{1-x_{c 1}}{0.75}\right)\left(\frac{1-y_{c 1}}{0.8}\right)} & \text { for } 0.75 \leq y_{c 1} \leq 1\end{cases}
$$

Using follower's optimal, the leader optimal can be calculated as:

$$
F_{l}^{1}\left(x_{c 1}, y_{c 1}\right)= \begin{cases}\frac{\left(\frac{x_{c 1}}{0.75}\right)\left(\frac{y_{c 1}-0.2}{0.2}\right)\left(-x_{c 1}+y_{c 1} / 2\right)+\left(\frac{x_{c 1}}{0.25}\right)\left(\frac{1-y_{c 1}}{0.8}\right)\left(y_{c 1}+x_{c 1}\right)}{\left(\frac{x_{c 1}}{0.75}\right)\left(\frac{y_{c 1}-0.2}{0.2}\right)+\left(\frac{x_{c 1}}{0.25}\right)\left(\frac{1-y_{c 1}}{0.8}\right)} & \text { for } 0.2 \leq x_{c 1} \leq 0.25 \\ \frac{\left(\frac{x_{c 1}}{0.75}\right)\left(\frac{y_{c 1}-0.2}{0.2}\right)\left(-x_{c 1}+y_{c 1} / 2\right)+\left(\frac{1-x_{c 1}}{0.75}\right)\left(\frac{1-y_{c 1}}{0.8}\right)\left(y_{c 1}+x_{c 1}\right)}{\left(\frac{x_{c 1}}{0.75}\right)\left(\frac{y_{c 1}-0.2}{0.2}\right)+\left(\frac{1-x_{c 1}}{0.75}\right)\left(\frac{1-y_{c 1}}{0.8}\right)} & \text { for } 0.25 \leq x_{c 1} \leq 0.4 \\ \frac{\left(\frac{x_{c 1}}{0.75}\right)\left(\frac{1-y_{c 1}}{0.6}\right)\left(-x_{c 1}+y_{c 1} / 2\right)+\left(\frac{1-x_{c 1}}{0.75}\right)\left(\frac{1-y_{c 1}}{0.8}\right)\left(y_{c 1}+x_{c 1}\right)}{\left(\frac{x_{c 1}}{0.75}\right)\left(\frac{1-y_{c 1}}{0.6}\right)+\left(\frac{1-x_{c 1}}{0.75}\right)\left(\frac{1-y_{c 1}}{0.8}\right)} & \text { for } 0.4 \leq x_{c 1} \leq 0.75 \\ \frac{\left(\frac{1-x_{c 1}}{0.25}\right)\left(\frac{1-y_{c 1}}{0.6}\right)\left(-x_{c 1}+y_{c 1} / 2\right)+\left(\frac{1-x_{c 1}}{0.75}\right)\left(\frac{1-y_{c 1}}{0.8}\right)\left(y_{c 1}+x_{c 1}\right)}{\left(\frac{1-x_{c 1}}{0.25}\right)\left(\frac{1-y_{c 1}}{0.6}\right)+\left(\frac{1-x_{c 1}}{0.75}\right)\left(\frac{1-y_{c 1}}{0.8}\right)} & \text { for } 0.75 \leq x_{c 1} \leq 1\end{cases}
$$


Stage 2 Problem:

Leader's fuzzy rules :

$\Re_{1}^{1}(x, y)$ : If $x_{2}$ is very high and $y_{2}$ is low then $\widetilde{f}_{l}^{2}\left(x_{2}, y_{2}\right)=x_{2}-y_{2}$

$\Re_{2}^{1}(x, y)$ : If $x_{2}$ is high and $y_{2}$ is high then $\widetilde{f}_{l}^{2}\left(x_{2}, y_{2}\right)=x_{2}+y_{2}$

where $y_{2}$ solves the follower's fuzzy rules by

$\Re_{1}^{1}(x, y)$ : If $x_{2}$ is very high and $y_{2}$ is low then $\widetilde{f}_{f}^{2}\left(x_{2}, y_{2}\right)=x_{2} / 2+y_{2}$

$\Re_{2}^{1}(x, y)$ : If $x_{2}$ is high and $y_{2}$ is high then $\widetilde{f}_{f}^{2}\left(x_{2}, y_{2}\right)=x_{2}-y_{2}$

$$
\begin{aligned}
& l_{1}^{2}= \begin{cases}\frac{1-x_{c 2}}{0.8} \cdot \frac{y_{c 2}-0.5}{0.1}, & \text { for } 0.5 \leq x_{c 2}, y_{c 2} \leq 0.6 \\
\frac{1-x_{c 2}}{0.8} \cdot \frac{1-y_{c 2}}{0.4}, & \text { for } 0.6 \leq x_{c 2}, y_{c 2} \leq 1\end{cases} \\
& l_{2}^{2}= \begin{cases}\frac{x_{c 2}-0.2}{0.6} \cdot \frac{y_{c 2}-0.5}{0.35}, & \text { for } 0.5 \leq x_{c 2}, y_{c 2} \leq 0.8 \\
\frac{1-x_{c 2}}{0.2} \cdot \frac{y_{c 2}-0.5}{0.35}, & \text { for } 0.8 \leq x_{c 2}, y_{c 2} \leq 0.85 \\
\frac{1-x_{c 2}}{0.2} \cdot \frac{1-y_{c 2}}{0.15}, & \text { for } 0.85 \leq x_{c 2}, y_{c 2} \leq 1\end{cases} \\
& F_{f}^{2}\left(x_{c 2}, y_{c 2}\right)= \begin{cases}\frac{\left(\frac{1-x_{c 2}}{0.8}\right)\left(\frac{y_{c 2}-0.5}{0.1}\right)\left(x_{c 2} / 2+y_{c 2}\right)+\left(\frac{x_{c 2}-0.2}{0.6}\right)\left(\frac{y_{c 2}-0.5}{0.35}\right)\left(x_{c 2}-y_{c 2}\right)}{\left(\frac{1-x_{c 2}}{0.8}\right)\left(\frac{y_{c 2}-0.5}{0.1}\right)+\left(\frac{x_{c 2}-0.2}{0.6}\right)\left(\frac{y_{c 2}-0.5}{0.35}\right)} & \text { for } 0.51 \leq y_{c 2} \leq 0.6 \\
\frac{\left(\frac{1-x_{c 2}}{0.8}\right)\left(\frac{1-y_{c 2}}{0.4}\right)\left(x_{c 2} / 2+y_{c 2}\right)+\left(\frac{x_{c 2}-0.2}{0.6}\right)\left(\frac{y_{c 2}-0.5}{0.35}\right)\left(x_{c 2}-y_{c 2}\right)}{\left(\frac{1-x_{c 2}}{0.8}\right)\left(\frac{1-y_{c 2}}{0.4}\right)+\left(\frac{x_{c 2}-0.2}{0.6}\right)\left(\frac{y_{c 2}-0.5}{0.35}\right)} & \text { for } 0.6 \leq y_{c 2} \leq 0.8 \\
\frac{\left(\frac{1-x_{c 2}}{0.8}\right)\left(\frac{1-y_{c 2}}{0.4}\right)\left(x_{c 2} / 2+y_{c 2}\right)+\left(\frac{1-x_{c 2}}{0.2}\right)\left(\frac{y_{c 2}-0.5}{0.35}\right)\left(x_{c 2}-y_{c 2}\right)}{\left(\frac{1-x_{c 2}}{0.8}\right)\left(\frac{1-y_{c 2}}{0.4}\right)+\left(\frac{1-x_{c 2}}{0.2}\right)\left(\frac{y_{c 2}-0.5}{0.35}\right)} & \text { for } 0.8 \leq y_{c 2} \leq 0.85 \\
\frac{\left(\frac{1-x_{c 2}}{0.8}\right)\left(\frac{1-y_{c 2}}{0.4}\right)\left(x_{c 2} / 2+y_{c 2}\right)+\left(\frac{1-x_{c 2}}{0.2}\right)\left(\frac{1-y_{c 2}}{0.15}\right)\left(x_{c 2}-y_{c 2}\right)}{\left(\frac{1-x_{c 2}}{0.8}\right)\left(\frac{1-y_{c 2}}{0.4}\right)+\left(\frac{1-x_{c 2}}{0.2}\right)\left(\frac{1-y_{c 2}}{0.15}\right)} & \text { for } 0.85 \leq y_{c 2} \leq 1\end{cases} \\
& F_{l}^{2}\left(x_{c 2}, y_{c 2}\right)= \begin{cases}\frac{\left(\frac{1-x_{c 2}}{0.8}\right)\left(\frac{y_{c 2}-0.5}{0.1}\right)\left(x_{c 2}-y_{c 2}\right)+\left(\frac{x_{c 2}-0.2}{0.6}\right)\left(\frac{y_{c 2}-0.5}{0.35}\right)\left(x_{c 2}+y_{c 2}\right)}{\left(\frac{1-x_{c 2}}{0.8}\right)\left(\frac{y_{c 2}-0.5}{0.1}\right)+\left(\frac{x_{c 2}-0.2}{0.6}\right)\left(\frac{y_{c 2}-0.5}{0.35}\right)} & \text { for } 0.51 \leq x_{c 2} \leq 0.6 \\
\frac{\left(\frac{1-x_{c 2}}{0.8}\right)\left(\frac{1-y_{c 2}}{0.4}\right)\left(x_{c 2}-y_{c 2}\right)+\left(\frac{x_{c 2}-0.2}{0.6}\right)\left(\frac{y_{c 2}-0.5}{0.35}\right)\left(x_{c 2}+y_{c 2}\right)}{\left(\frac{1-x_{c 2}}{0.8}\right)\left(\frac{1-y_{c 2}}{0.4}\right)+\left(\frac{x_{c 2}-0.2}{0.6}\right)\left(\frac{y_{c 2}-0.5}{0.35}\right)} & \text { for } 0.6 \leq y_{c 2} \leq 0.8 \\
\frac{\left(\frac{1-x_{c 2}}{0.8}\right)\left(\frac{1-y_{c 2}}{0.4}\right)\left(x_{c 2}-y_{c 2}\right)+\left(\frac{1-x_{c 2}}{0.2}\right)\left(\frac{y_{c 2}-0.5}{0.35}\right)\left(x_{c 2}+y_{c 2}\right)}{\left(\frac{1-x_{c 2}}{0.8}\right)\left(\frac{1-y_{c 2}}{0.4}\right)+\left(\frac{1-x_{c 2}}{0.2}\right)\left(\frac{y_{c 2}-0.5}{0.35}\right)} & \text { for } 0.8 \leq y_{c 2} \leq 0.85 \\
\frac{\left(\frac{1-x_{c 2}}{0.8}\right)\left(\frac{1-y_{c 2}}{0.4}\right)\left(x_{c 2}-y_{c 2}\right)+\left(\frac{1-x_{c 2}}{0.2}\right)\left(\frac{1-y_{c 2}}{0.15}\right)\left(x_{c 2}+y_{c 2}\right)}{\left(\frac{1-x_{c 2}}{0.8}\right)\left(\frac{1-y_{c 2}}{0.4}\right)+\left(\frac{1-x_{c 2}}{0.2}\right)\left(\frac{1-y_{c 2}}{0.15}\right)} & \text { for } 0.85 \leq y_{c 2} \leq 1\end{cases}
\end{aligned}
$$


By using Lingo, we get the solution as $F_{l}^{2}=7.0776$ and $F_{f}^{2}=2.657$ at $x_{c 2}=0.6$ and $y_{c 2}=0.8$.

\section{Conclusion}

In a two level structure, to built a mathematical model, decision makers need deterministic type information. Where both the decision maker have their own objective function to optimize under some crisp inequalities. If the information source from where the crisp inequalities has to be built, is not known precisely i.e we know the linguistic relationship between the objective function and the constraints. In that case it is difficult to build a deterministic bi-level programming model. Here, we present a fuzzy rule-base bi-level programming model which overcomes this difficulty.

To make this long article short, we conclude here that, In a hierarchical administrative structure, To solve a fuzzy rule-based bi-level programming problem where the deterministic functional relationship between the objective functions of decision makers and their decision space is not known exactly, a bi-level programming with fuzzy rule base has been constructed. In order to solve the problem, a suitable fuzzy inference scheme and dynamic programming approach has been used to convert fuzzy rule-base bi-level programming problem into crisp bi-level programming problem of two variables. A multi-stage decision making approach has been used to find the solution of the problem.

\section{Acknowledgement}

We would like to thank the journal editor and the anonymous referees for their constructive comments and suggestions that have helped to improve this paper.

\section{References}

[1] Agarwal, R. and Mittal. M. (2019). Inventory Classification Using Multi-Level Association Rule Mining. International Journal of Decision Support System Technology, 11(2), 1-12. doi: 10.4018/ijdsst.2019040101

[2] Amat, F. J. and McCarl, B. (1981). A representation and economic interpretation of a twolevel programming problem. Journal of the Operational Research Society, 32(9), 783-792. doi: $10.1057 /$ jors.1981.156

[3] Bard, J. F. (1984). Optimality conditions for the bilevel programming problem. Naval Research Logistics Quarterly, 31(1), 13-26. doi: 10.1002/nav.3800310104

[4] Bard, J. F. (1991). Some properties of the bilevel programming problem. Journal of Optimization Theory and Applications, 68(2), 371-378. doi: 10.1007/bf00941574

[5] Bialas, W. F. and Karwan, M. H. (1984). Two-level linear programming. Management Science, 30(8), 1004-1020. doi: 10.1287/mnsc.30.8.1004

[6] Candler, W. and Townsley, R. (1982). A linear two-level programming problem. Computers and Operations Research, 9(1), 59-76. doi: 10.1016/0305-0548(82)90006-5

[7] Chakraborty, D. and Guha, D. (2013). Multi-objective optimization based on fuzzy if-then rules. IEEE Proceedings of International Conference on Fuzzy Systems. doi: 10.1109/fuzzieee.2013.6622519

[8] Gao, Y., Zhang, G., Ma, J. and Lu, J. (2010). A $\lambda$-Cut and Goal-Programming-Based Algorithm for Fuzzy-Linear Multiple-Objective Bilevel Optimization. EEE Transactions on Fuzzy Systems, 18(1), 1-13. doi: 10.1109/tfuzz.2009.2030329

[9] Jankovic, M. and Zarate, P. (2011). Discrepancies and Analogies in Artificial Intelligence and Engineering Design Approaches in Addressing Collaborative Decision-Making, International Journal of Decision Support System Technology, 3(2), 1-14. doi: 10.4018/jdsst.2011040101 
[10] Kosuch, S., Le Bodic, P., Leung, J. and Lisser, A. (2012). On a stochastic bilevel programming problem. Networks, 59(1), 107-116. doi: 10.1002/net.20482

[11] Nishizaki, I., Masatoshi S., Kato, K. and Katagiri, H. (2003). Stackelberg Solutions to Two-Level Linear Programming Problems with Random Variable Coefficients. Multi-Objective Programming and Goal Programming, 209-214. Springer: Berlin. doi: 10.1007/978-3-540-36510-5_29

[12] Masatoshi, S., Nishizaki, I. and Uemura, Y. (2000). Interactive fuzzy programming for multi-level linear programming problems with fuzzy parameters. Fuzzy Sets and Systems, 109(1), 3-19. doi: 10.1016/s0165-0114(98)00130-4

[13] Shi, C., Lu, J. and Zhang, G. (2005). An extended Kuhn-Tucker approach for linear bilevel programming. Applied Mathematics and Computation, 162(1), 51-63 doi: 10.1016/j.amc.2003.12.089

[14] Singh, V. P., and Chakraborty, D. (2015). A Dynamic Programming Algorithm for Solving BiObjective Fuzzy Knapsack Problem. Mathematics and Computing, 289-306. Springer: New Delhi. doi: 10.1007/978-81-322-2452-5_20

[15] Singh, V. P. and Chakraborty, D. (2017). Solving bi-level programming problem with fuzzy random variable coefficients. Journal of Intelligent and Fuzzy Systems, 32(1), 521-528. doi: 10.3233/jifs152354

[16] Takagi, T. and Sugeno, M. (1985). Fuzzy identification of systems and its applications to modeling and control. IEEE Transactions on Systems, Man, and Cybernetics, 15(1), 116-132. doi: 10.1109/tsmc.1985.6313399

[17] Wen, U. P. and Hsu, S. T. (1991). Linear bi-level programming problems - A review. Journal of the Operational Research Society, 42(2), 125-133. doi: 10.1038/sj/jors/0420204

[18] Wen, U. P. and Hsu, S. T. (1992). Efficient solutions for the linear bilevel programming problem. European Journal of Operational Research, 62(3), 354-362. doi: 10.1016/0377-2217(92)90124-r

[19] Zadeh, L. A. (1975). The concept of a linguistic variable and its application to approximate reasoning-I. Information Sciences, 8(3), 199-249. doi: 10.1016/0020-0255(75)90036-5

[20] Zarour, K. and Benmerzoug, D. (2019). A Decision-Making Support for Business Process Outsourcing to a Multi-Cloud Environment. International Journal of Decision Support System Technology, 11(1), 66-92. doi: 10.4018/ijdsst.2019010104

[21] Zhang, G. and Lu, J. (2013). Fuzzy bilevel programming with multiple objectives and cooperative multiple followers. Journal of Global Optimization, 47(3), 403-419. doi: /10.1007/s10898-0089365-Z

[22] Zhou, G., Zhao, R. and Zhou, Y. (2018). Solving large-scale 0-1 knapsack problem by the socialspider optimization algorithm. International Journal of Computing Science and Mathematics, 9(5), 433-441. doi: 10.1504/ijcsm.2018.095497 